\title{
Atrial Fibrillation and Systemic Thromboembolism - Causal Correlation or a Message of Atrial Disease?
}

\author{
Fibrilação Atrial e Tromboembolismo Sistêmico - Relação Causal ou uma \\ Mensagem de Doença Atrial?
}

J. Tarcísio Medeiros de Vasconcelos ${ }^{1, *}$, Carlos Eduardo Duarte1, Silas dos Santos Galvão Filho

ORCID IDs

Vasconcelos JTM (D) https://orcid.org/0000-0002-5152-2648

Duarte CE (D) https://orcid.org/0000-0001-6671-0820

Galvão Filho SS (D https://orcid.org/0000-0001-5236-164X

\section{INTRODUCTION}

The correlation between atrial fibrillation (AF) and thromboembolism is well known. In 1951, Raymond Daley et al. associated the occurrence of this arrhythmia to systemic embolic events with consistent information in patients with chronic rheumatic heart disease ${ }^{1}$. In this study involving 194 patients with rheumatic heart disease who were victims of thromboembolism, with autopsy information in 39 patients, the presence of AF was demonstrated in about $90 \%$ of cases. The classic Framingham study, published in 1978, was the first large study that established this same correlation in nonrheumatic individuals, showing that individuals with AF are nearly six times more likely to have a stroke than the AF-free individuals with characteristics adjusted for sex, age and blood pressure ${ }^{2}$. The inclusion of rheumatic individuals has raised this risk to approximately 18 times. Since this important publication, several studies have corroborated these findings ${ }^{3-7}$.

Table 1 presents the results of five studies involving more than 100,000 patients, which locate the prevalence of $\mathrm{AF}$ in ischemic stroke patients around 15 to $40 \%$. It is quite reasonable, however, to deduce that the prevalence of arrhythmia is higher even in 60 to $85 \%$ of patients with ischemic stroke without prior AF diagnosis, considering the possibility of undiagnosed asymptomatic arrhythmia. In a systematic review and meta-analysis of 32 studies involving approximately 5,000 patients with no history of AF who suffered an ischemic stroke submitted to aggressive heart rate monitoring by Holter $24 \mathrm{~h}$, Holter 7 days or implantable event monitor, the diagnosis of $\mathrm{AF}$ was demonstrated in about $11 \%$ of patients ${ }^{8}$. Therefore, based on the information obtained in these extensive studies over the last six decades, with incorporated results of significant strength and consistency of association, the following paradigm was constructed: "atrial fibrillation causes thromboembolic ischemic stroke". 
Table 1. Results of five different studies that together include more than 100,000 patients that characterize the high frequency of atrial fibrillation in ischemic stroke patients.

\begin{tabular}{cccc}
\hline Author & Year & $\begin{array}{c}\text { Ischemic stroke } \\
(\mathrm{n})\end{array}$ & $\begin{array}{c}\text { Atrial fibrillation } \\
(\%)\end{array}$ \\
\hline Wolf PA et al. & 1987 & 462 & 14.7 \\
Thygesen SK et al. $^{3}$ & 2009 & 3,849 & 19.2 \\
Hannon N et al. ${ }^{5}$ & 2010 & 568 & 31.2 \\
Björck S et al. & 2013 & 4,565 & 38 \\
Friberg L et al. & 2014 & 94,083 & 33.4 \\
\hline
\end{tabular}

\section{ATRIAL FIBRILLATION AND ATRIAL DISEASE}

Atrial fibrillation identified as an independent risk factor for ischemic stroke has become a cornerstone of anticoagulant therapy, with consistently positive results when other risk stratification elements are added, which involved the construction of risk scores, now universally used for therapeutic decision-making, such as $\mathrm{CHADS}_{2}$, $\mathrm{CHA}_{2} \mathrm{DS}_{2}-\mathrm{VASc}$, and ATRIA ${ }^{9-11}$. It is interesting to reflect on the risk conditioning of AF for ischemic stroke to the aggregation of other parallel elements to catalyze this risk, such as presence or absence of heart failure, hypertension, diabetes, advanced age, and valve disease. It is also interesting to note that these same risk elements for ischemic stroke occurrence in patients with $\mathrm{AF}$ are also identified as indicative risk elements for the occurrence of future AF in patients without documented AF.

In a large Swedish population study, the prevalence of $\mathrm{AF}$ in patients with a $\mathrm{CHA}_{2} \mathrm{DS}_{2}-\mathrm{VASc}$ score equal to zero was slightly higher than $10 \%$, reaching values close to $70 \%$ when this score reached a value equal to nine ${ }^{7}$. Why does isolated AF have a small probability of risk for ischemic stroke compared to that associated with diabetes and hypertension, for example? Would AF be a direct determinant of ischemic stroke? Or is it just the expression of a differentiated cardioembolic risk linked to an underlying thrombogenic baseline condition? In response to these questions, data indicate that the use of the $\mathrm{CHA}_{2} \mathrm{DS}_{2}$-VASc score identifies the risk of thromboembolism also from individuals in sinus rhythm. In a large Danish study involving more than 136,000 individuals with heart failure in sinus rhythm, the use of this score was able to identify in a very predictable way the thromboembolic risk in the different stratification categories ${ }^{12}$. In another community study conducted in Trieste, Italy, in which nearly 12,500 hypertensive individuals without use of anticoagulant therapy were included, with about 11,000 individuals in sinus rhythm and 1,500 with atrial fibrillation, the $\mathrm{CHA}_{2} \mathrm{DS}_{2}-\mathrm{VASc}$ score also identified thromboembolic risk in the different stratification categories in both groups of patients; surprisingly, the ability to identify thromboembolic risk in patients with a score of six or more was equivalent among patients with atrial fibrillation and sinus rhythm ${ }^{13}$. Therefore, elements are indicating that a score initially developed and validated to stratify the risk of thromboembolism in patients with atrial fibrillation is also capable of identifying the risk of future development of AF itself and the thromboembolic risk in individuals with stable sinus rhythm, that is, without AF.

The unfolding of the $\mathrm{CHA}_{2} \mathrm{DS}_{2}$-VASc score in its various components allows the development of reasoning that places the findings described within a biological plausibility model. Cardiopathy, heart failure, hypertension, advanced age, diabetes, and vascular disease are determining factors or markers of atrial myocardial aggression. Atrial myocardial aggression is potentially thrombogenic being determinant, and/or catalyst of AF itself, which in turn feeds back the myocardial aggression itself (Figs. 1 and 2). Thus, the concept of atrial fibrotic cardiopathy is approached.

The characterization of atrial fibrotic cardiopathy was initially conducted in 2012 by Kottkamp, which drew attention to patients who had structural and electrophysiological impairment of the atrial musculature in the absence of underlying diseases that could justify them ${ }^{15}$. Figure 3 corresponds to the voltage maps of the right and 


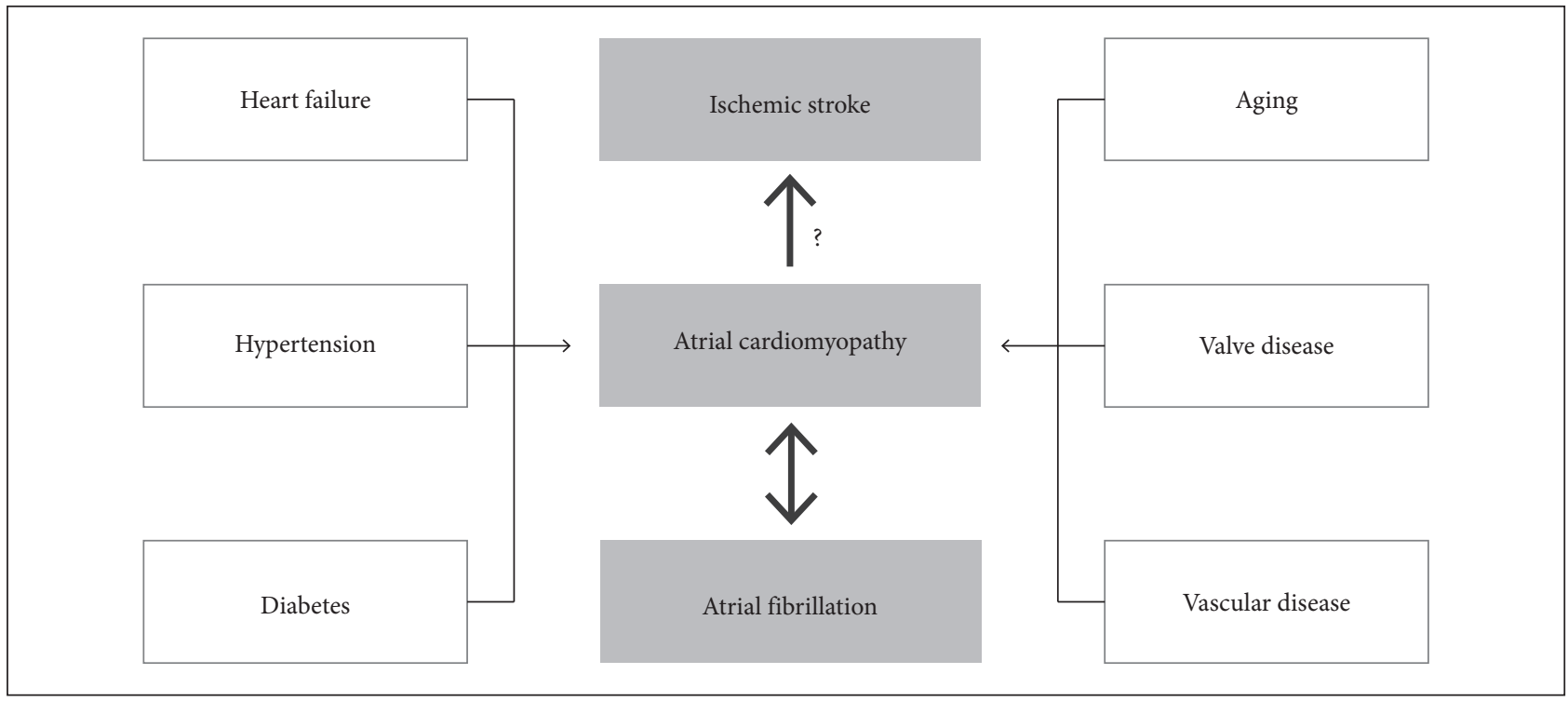

Figure 1. Potential correlation of thromboembolic risk markers of atrial fibrillation with impairment of the structural and electrical atrial itself. Conditions such as heart failure, hypertension, diabetes, aging, valve, and vascular disease are, in fact, potential determinants of structural aggression to the atrial myocardium, implying atrial cardiomyopathy. Atrial cardiomyopathy is a determinant of atrial fibrillation, which in turn enhances the process of atrial myocardial aggression. The cardioembolic risk would be caused not by direct atrial fibrillation (which may be absent), but by established atrial disease.

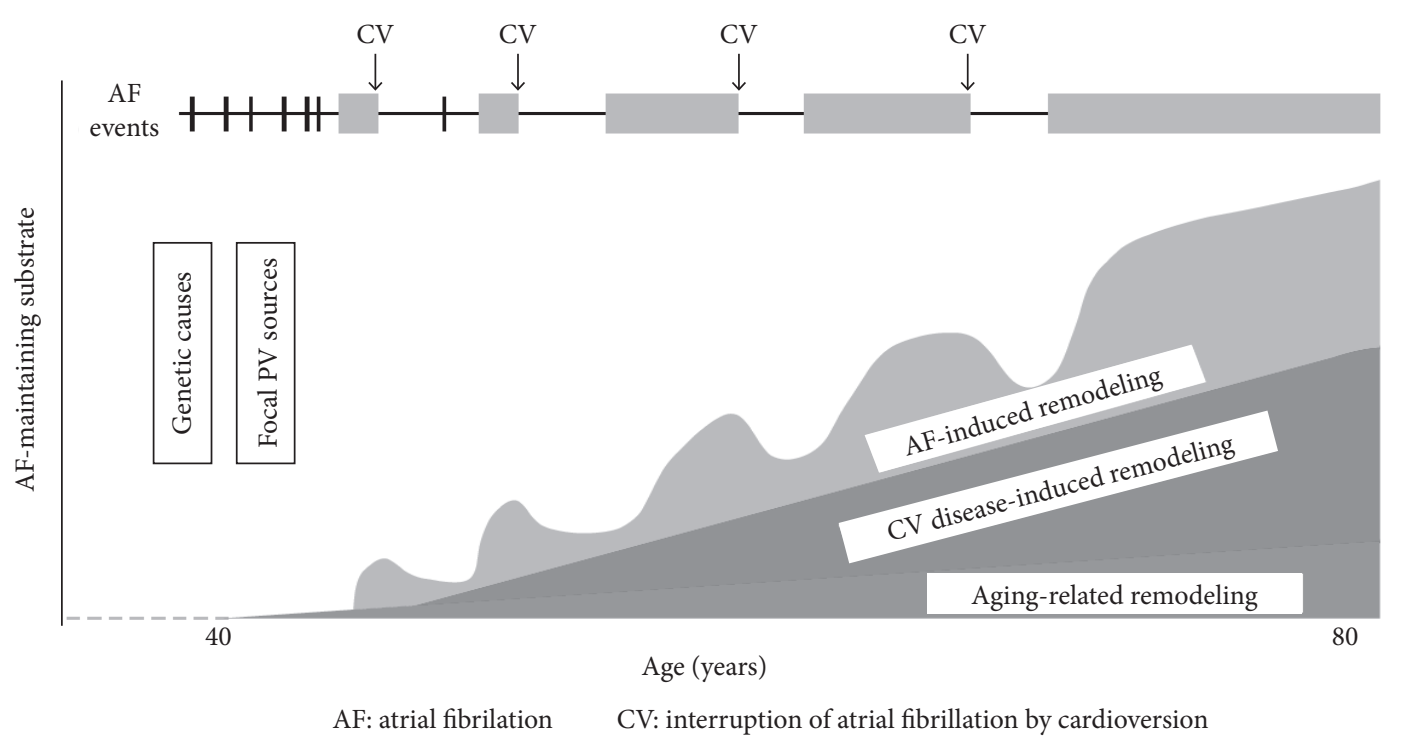

Figure 2. The interrelation of determining factors of electrical and structural atrial remodeling. Atrial fibrillation may be primary linked to genetic factors and pulmonary triggers, or it may be secondary to progressive atrial disease itself as a result of aging and/ or muscle impairment secondary to comorbidities. There is a combination scenario of factors whose final result is atrial fibrotic cardiomyopathy. Adapted from Guichard and Nattel ${ }^{14}$.

left atria obtained from a 42-year-old woman with no stable structural heart disease, with "solitary" tachycardia and atrial fibrillation. The images were obtained during the electrophysiological study and percutaneous ablation to treat these arrhythmias. The grey areas correspond to the low-voltage regions, indicative of the presence of a scar, and the purple areas correspond to the regions of normal myocardium. Extensive scars are noted along the walls of both atria, with normal myocardium in restricted areas, indicating significant biatrial fibrotic impairment. In such a circumstance, even in the case of a patient with low cardioembolic risk by standard criteria $\left(\mathrm{CHA}_{2} \mathrm{DS}_{2}\right.$ VASC $=0$ ), it is natural to question the permanent use of anticoagulant therapy, regardless of whether or not stable sinus rhythm is present. 


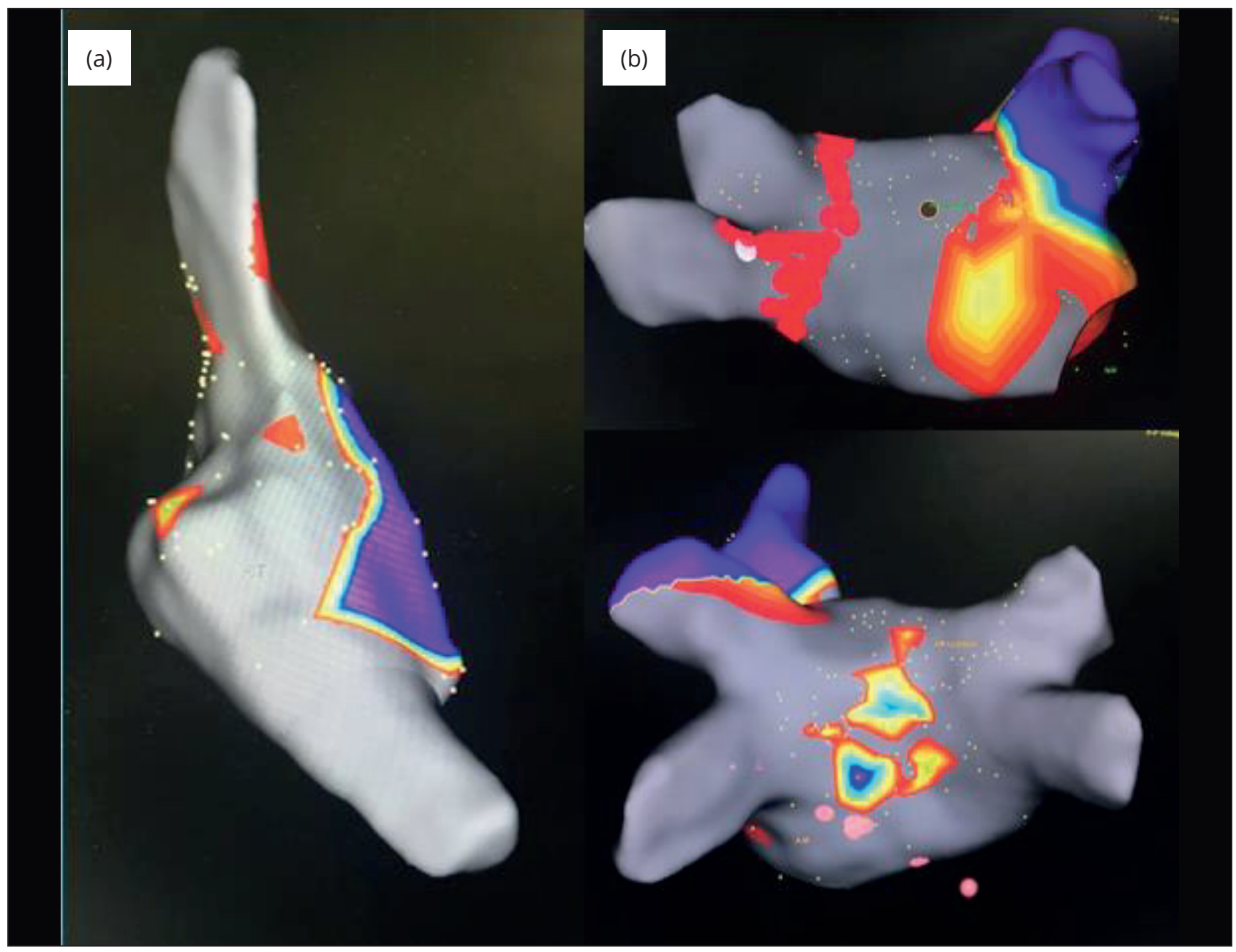

Figure 3. Voltage maps of the right (a) and left (b) atria constructed during an ablation procedure.

In an excellent consensus document prepared by four international medical societies, in which a significant review on the subject was carried out, published in 2016, atrial fibrotic cardiomyopathy was classified into four types according to the histological impairment pattern, related to different types of etiological factors that determine muscle aggression ${ }^{16}$. The concept and characterization of the disease became more comprehensive and detailed in this document, not restricted to atrial involvement dissociated from the identification of systemic diseases, but included within the universe of all diseases that may generate some form of atrial myocardial aggression, including aging, obesity, hypertension, myocarditis, heart failure, diabetes, deposit diseases, hereditary diseases, remodeling induced by arrhythmia itself, etc. The fundamental question is whether the presence of atrial structural impairment is not the primary determinant of increased cardioembolic risk since atrial fibrillation is a simple marker of the presence of disease and/or a mere aggravating of this cardioembolic risk - different information points in that direction. The presence of atrial dilation has been identified as a risk factor for ischemic stroke, even in the absence of diagnosed atrial fibrillation ${ }^{17}$. The presence of atrial fibrosis diagnosed by late enhancement technique on magnetic resonance imaging (MRI) in some studies was related to a history of ischemic stroke, presence of thrombus in left atrial appendage, and spontaneous contrast in left atrium diagnosed by transesophageal echocardiography ${ }^{18-20}$. The diagnosis of atrial fibrosis by voltage map in ablation procedures for the treatment of atrial fibrillation was related to a higher probability of preexisting ablation, the occurrence of ischemic stroke and silent brain damage diagnosed by MRI ${ }^{21}$. 


\section{STROKE AND HEART RHYTHM: ELECTROCARDIOGRAPHIC DOCUMENTATION OF THE EVENT}

The ideal scenario to establish a clear cause and effect correlation between atrial fibrillation and the cardioembolic event is the recording of the cardioembolic event itself through continuous monitoring of cardiac rhythm, especially in the period preceding its occurrence. The natural challenge to obtain this information is the availability of cardiac monitoring before and during the moment of the cardioembolic event. The underlying heart rhythm records in victims of a thromboembolic accident usually happen after its occurrence, situations in which the presence of stable sinus rhythm does not mean normal rhythm in the moments, days, or weeks preceding the event itself. It is plausible to imagine a sequence of facts initiated by the occurrence of atrial fibrillation precipitating the formation of a left atrial thrombus, its displacement generating distant embolic complications and spontaneous conversion of the atrial fibrillation to sinusal rhythm, in such a way that, on the occasion of the first medical care, the presence of sinusal rhythm is verified.

The possibility of monitoring heart rhythm by ultra-long duration with implantable devices opened a new perspective of investigation that allows a deepening in this subject. Stimulation cardiac devices, including pacemakers, cardioverter-defibrillators, and cardiac resynchronizers are prostheses that possess sophisticated resources of permanent monitoring of cardiac rhythm and storage of this information, with the advantage of allowing the recording of intracardiac atrial and ventricular events, thus aggregating invaluable diagnostic precision (Fig. 4).

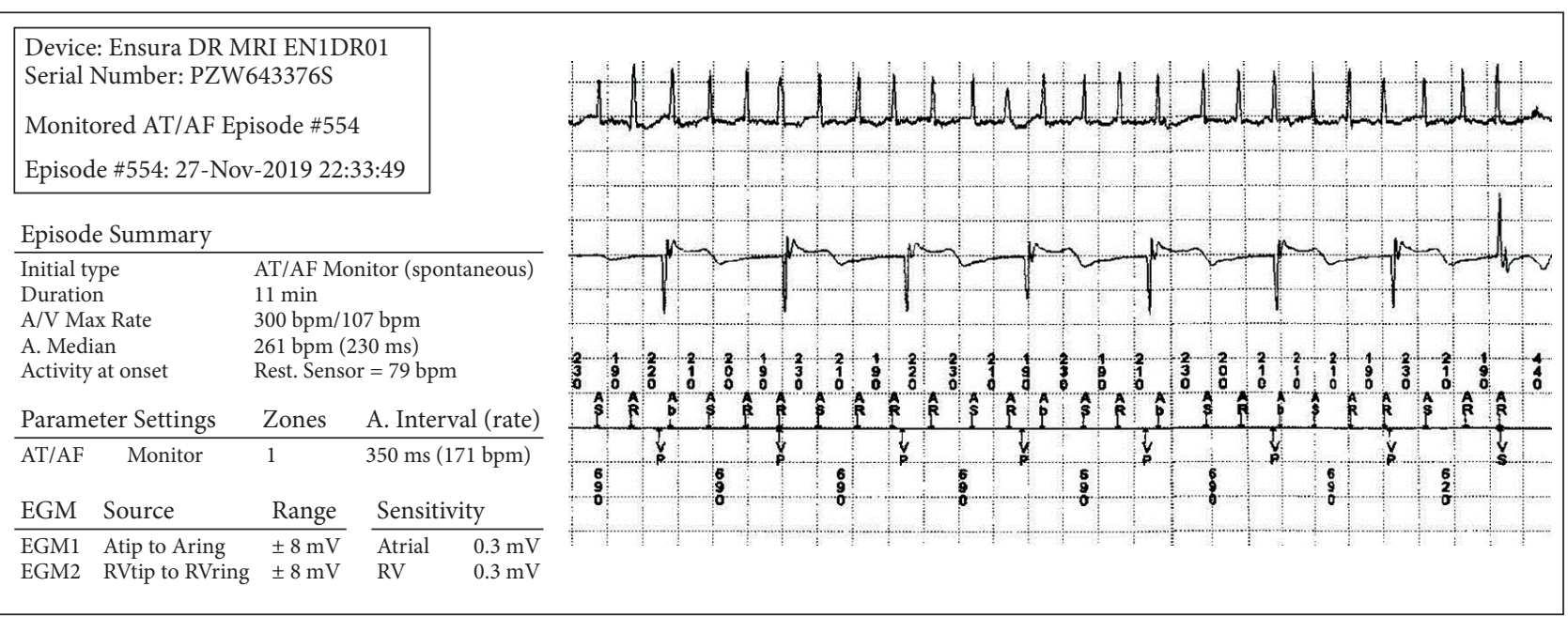

Figure 4. 11-minute subclinical primary atrial tachycardia episode detected by a double chamber pacemaker. The first channel corresponds to the atrial electrograms, where atrial tachycardia with a frequency close to $300 \mathrm{bpm}$ is evident. The second channel corresponds to ventricular electrograms that demonstrate that the ventricles are under pacemaker stimulation at the moment of tachycardia due to an atrioventricular block. The lower channel represents the identification in marks of how the device is interpreting the underlying heart rhythm.

Some studies have taken advantage of these technological resources in order to more consistently evaluate the likely interrelations between the occurrence of atrial tachycardias and thromboembolic events from a time perspective, with intriguing results. The TRENDS Study ${ }^{22}$, published in 2009, was a prospective observational study that recruited patients with pacing/defibrillation devices, with one or more cardioembolic risk factors (heart failure, hypertension, diabetes, age 65 or older, history of thromboembolic accident), to be followed for an extended period trying to identify thromboembolic events in this follow-up and establish its correlation with the underlying cardiac rhythm. The objective of the study was actually to determine whether or not brief subclinical episodes of atrial tachycardias (often detected in routine device evaluations) would be related to a differentiated cardioembolic 
risk. The premise would be that patients with higher atrial tachycardia burden, i.e., with more atrial tachycardia in the period (hours in atrial tachycardia per day) could correspond to a subpopulation with higher thromboembolic risk. Relevant atrial tachycardias were those with duration equal to or greater than 20 seconds. Although the non-inclusion of anticoagulated patients was foreseen in the original study design, all patients were included in the final evaluation, probably due to restrictions in the sample size combined with a low final rate of outcomes, which would imply a limitation in the valorization of results. Nearly 2,500 patients were included in the study, with a follow-up of about 1.5 years. The $\mathrm{CHADS}_{2}$ score average was 2.2 , generating an annualized risk of more than $4 \%$ for systemic thromboembolism. Forty outcomes occurred in the follow-up, generating an annualized rate of events of only $1.2 \%$, lower than expected for a population with atrial fibrillation clinically diagnosed with similar risk expectations. A direct relation of the atrial tachycardia burden and the occurrence of systemic thromboembolism was demonstrated; patients with a burden equal to or greater than $5.5 \mathrm{~h}$ (corresponding to the sum of all atrial tachycardia duration that occurred on at least one day in a previous 30-day period of continuous monitoring) were about twice as likely to present an event as those with a zero burden. In other words, the higher the number and/ or duration of atrial tachycardias, the higher the incidence of outcomes. However, an unexpected time-lapse was demonstrated between the occurrence of atrial tachycardia and the thromboembolic event: only about $28 \%$ of the patients who were victims of an event had some type of atrial tachycardia in the 30 days before this event ${ }^{23}$.

A more recent study, the ASSERT (Asymptomatic Atrial Fibrillation and Stroke Evaluation in Pacemaker Patients and the Atrial Fibrillation Reduction Atrial Pacing Trial) ${ }^{24}$ brought similar results. Approximately 2,600 patients with pacemakers or cardioverter-defibrillators, with no history of known atrial fibrillation, aged 65 years or older, without the use of anticoagulants, were followed up in order to assess the temporal correlation between the occurrence of atrial tachycardias and thromboembolic events. Atrial tachycardias were defined by their occurrence detected in devices with a duration longer than 6 minutes. After a mean follow-up of 30 months, 51 patients were victims of potentially cerebral and systemic cardioembolic events, in which atrial tachycardias lasting more than 6 minutes were identified in 26 cases (51\%). However, the occurrence of this atrial tachycardia occurred in only four patients (8\%) in the 30 days preceding the cardioembolic event.

Another multicenter study, the IMPACT study ${ }^{25}$, evaluated the role of occasional anticoagulation in the prevention of thromboembolic accidents, based on the detection of atrial tachycardias by implantable cardioverter-defibrillators. The detection of atrial tachycardias by the devices was quickly notified to the attending physician by a permanent remote monitoring system, enabling the intermittent administration of anticoagulant drugs. Vitamin $\mathrm{K}$ antagonists and direct-acting anticoagulants were used. The hypothesis was that intermittent anticoagulation used only after the diagnosis of atrial tachycardias would be a superior strategy to permanent anticoagulation based on standard criteria, in the reduction of an outcome composed of stroke, systemic embolism, and significant bleeding. Atrial tachycardias were considered relevant if 36 or more atrial cycles out of 48 were more than $200 \mathrm{bpm}$. Patients with permanent atrial fibrillation were excluded. It was a prospective study in which approximately 2,700 patients were randomized for intermittent anticoagulation or the control group. Anticoagulation in the control group patients was permanently instituted if atrial fibrillation was detected in the periodic clinical evaluations, either by conventional methods or by the interrogation of the devices themselves. The study was expected to last 36 months but was interrupted prematurely at 24 months because intermittent anticoagulation could not be shown to be superior. Sixty-nine thromboembolic events were documented in the entire population, in 58\% (40 events) in the absence of atrial tachycardias detected throughout the follow-up. Of the 29 patients in whom atrial tachycardias were recorded, in 20, they occurred before the thromboembolic accident, of which only 6 (8.7\%) occurred at the time or in the 30 days before the thromboembolic accident. Once again, the absence of a clear temporal correlation between the arrhythmic event and the thromboembolic event was demonstrated.

The results of these three studies are graphically represented in Fig. 5.

A recent meta-analysis, published in 2018, included 11 studies that evaluated the diagnosis and significance of subclinical atrial tachycardias in patients with implantable electronic heart devices ${ }^{26}$. Subclinical atrial fibrillation-type 


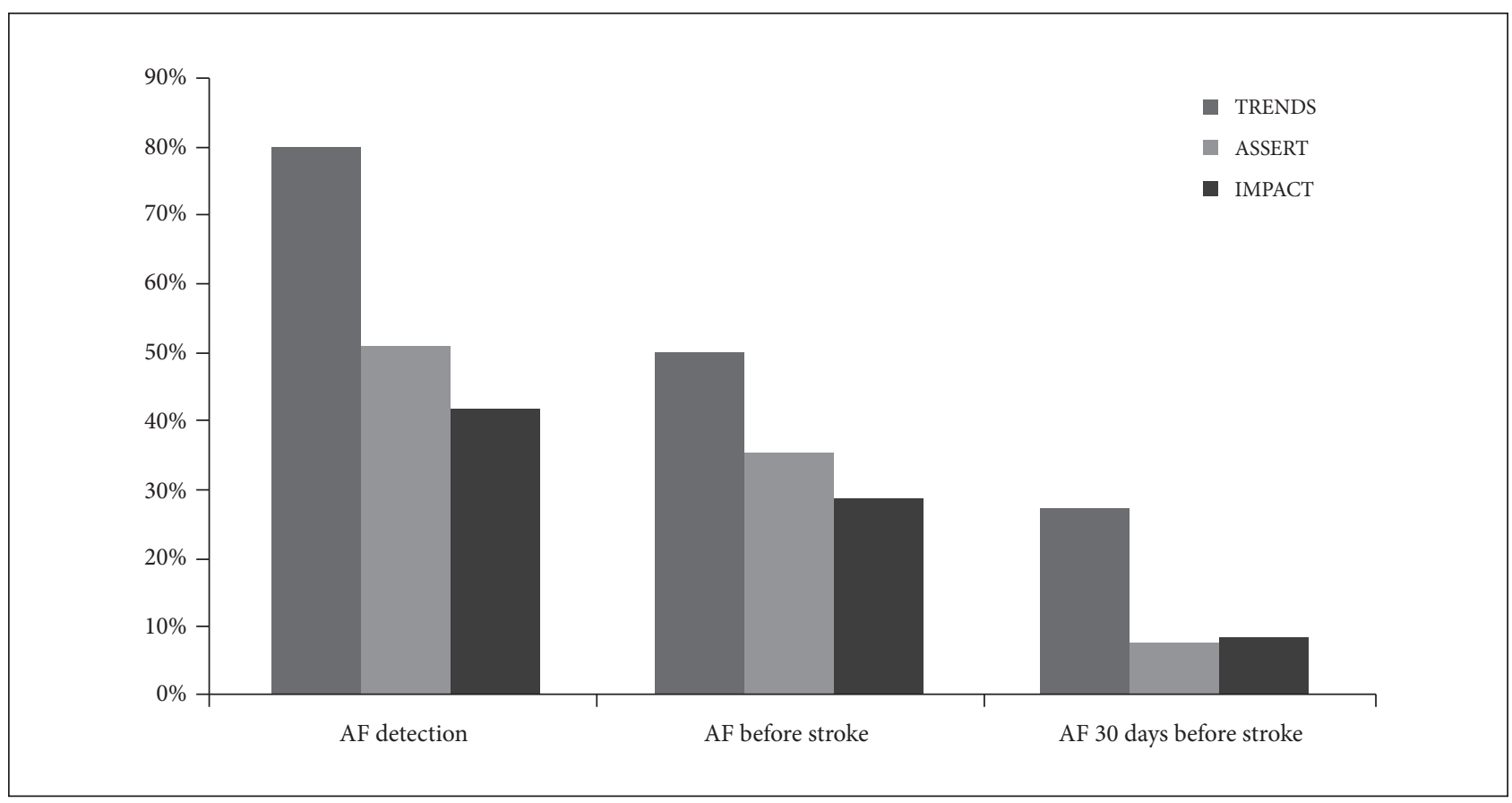

Figure 5. Graphical presentation of TRENDS, ASSERT and IMPACT study results. The information is derived from patients who suffered cardioembolic accidents, predominantly ischemic strokes, while they were under permanent cardiac rhythm monitoring. The left columns represent the patients who, at some point in the monitoring, presented atrial tachycardias. It is noteworthy that 40 to $80 \%$ of patients had tachycardia recorded. The middle columns represent the patients who presented atrial tachycardia before the embolic accident, varying between 30 and 50\%. The right columns represent the patients who presented atrial tachycardias in the 30 days preceding the embolic event. It is noteworthy that that number does not reach $30 \%$.

tachycardias were identified in $35 \%$ of patients. The presence of subclinical atrial fibrillation was strongly related to the occurrence of clinical atrial fibrillation with a risk of about six times higher. Only $17 \%$ of ischemic strokes occurred during atrial fibrillation, and only $29 \%$ of patients who had a cardioembolic accident had atrial fibrillation recorded by the devices in the 30 days prior to the event.

This set of information indicates that despite the presence of atrial tachycardias, especially atrial fibrillation, being a risk marker for the occurrence of thromboembolic phenomena, its occurrence would not be the direct cause of the event in patients with silent atrial tachycardias detected in pacing and/or cardiac defibrillation devices. However, data derived from these studies should be viewed with caution due to several factors: 1) these studies involved a specific subpopulation of patients, wearers of pacemakers or cardioverter-defibrillators implanted because of particular and specific clinical conditions, and these conclusions cannot be transferred to the entire set of individuals with atrial fibrillation; 2) the atrial tachycardias diagnosed in these studies, including atrial fibrillation itself, generally corresponded to subclinical, self-limited tachycardias, most of them of short duration, whose significance is potentially different from that observed in patients classically involved in extensive studies that identified atrial fibrillation as a major villain - one of the most emblematic elements of this finding corresponds to the fact that the rates of global events in these patients was characteristically lower than that expected for patients with clinically diagnosed fibrillation, considering the risk classes by $\mathrm{CHADS}_{2}$ or $\mathrm{CHA}_{2} \mathrm{DS}_{2}-\mathrm{VASC}$ scores; 3 ) the very diagnostic characteristics of implantable electronic cardiac devices must always be considered, which are fallible, as a consequence of a diversity of factors, such as "under or oversensing" (Figure 6) or the actual (predetermined) way of programming to identify tachycardias - in short, devices can misdiagnose tachycardias that did not exist or fail to diagnose tachycardias that did occur. 


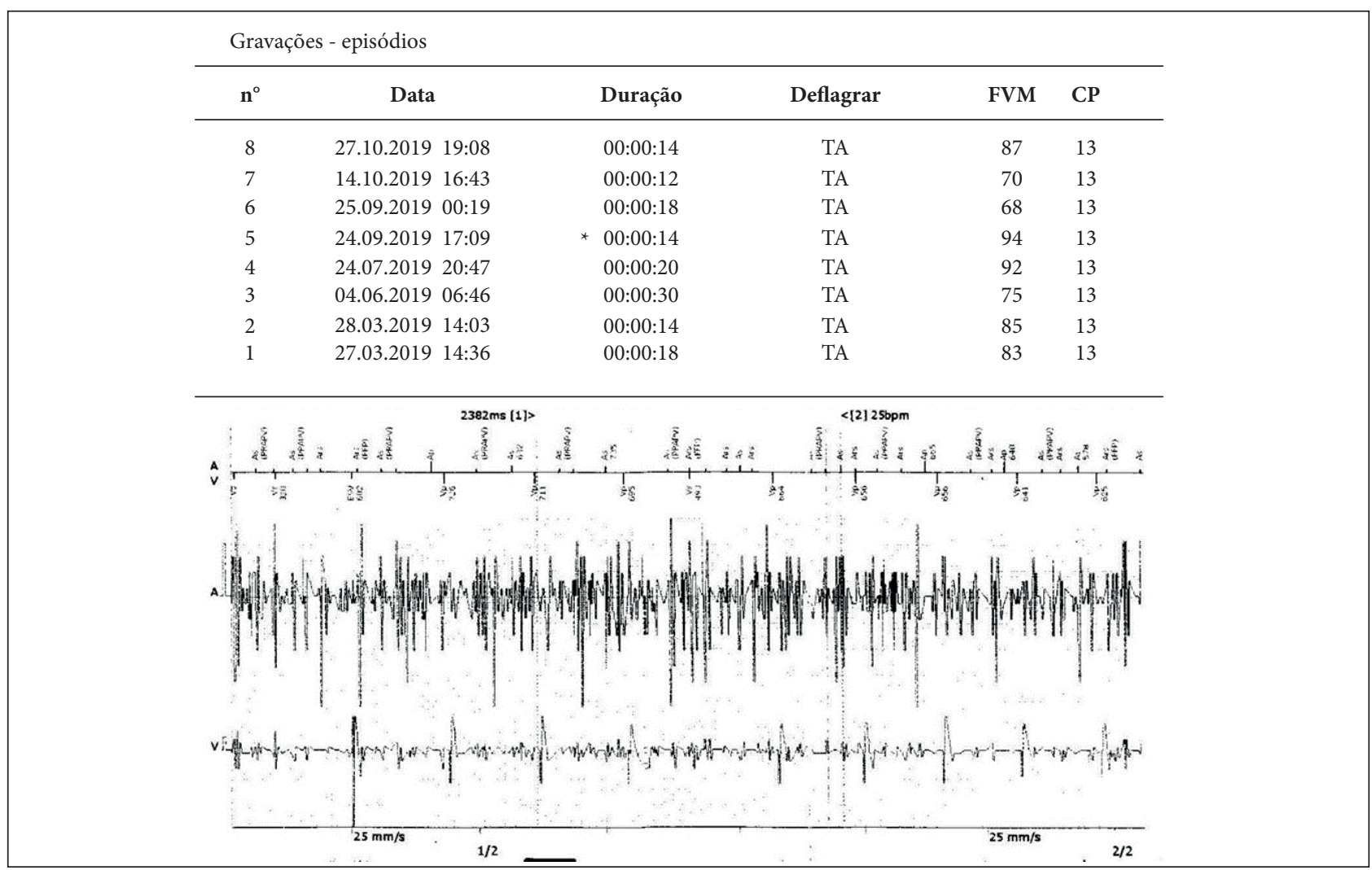

Figure 6. Report obtained from a device evaluation that informs the occurrence of several episodes of atrial tachycardia. The analysis of intracardiac electrograms, however, demonstrates the presence of electrical artifacts in the atrial and ventricular channels corresponding to electrical noises, possibly resulting from incomplete fractures of the electrodes. It is, therefore, a false atrial tachycardia.

\section{WHERE ARE WE GOING?}

In light of all the above considerations, what is characterized at the very least is that sick atria "fibrillate", fibrillation produces or aggravates atrial disease; that classically indicated risk factors for thromboembolism in atrial fibrillation are also (or before that) risk factors for the development or presence of atrial disease and the very occurrence of future atrial fibrillation; and that the direct cause and effect correlation between atrial fibrillation and the punctual occurrence of the embolic event is doubtful. Under the diagnostic aspect, the most aggressive investigation scenario of patients is opened, either in sinusal rhythm or atrial fibrillation, in order to identify elements that allow the recognition of the presence of atrial fibrotic cardiopathy. Under the prophylactic and therapeutic aspects, the scenario of preventing atrial disease by addressing the peripheral determinant conditions opens up, treating primarily the atrial disease established by the treatment of the determinant diseases, maintaining sinus rhythm to avoid aggravating the atrial disease itself and broadening the perspective of cardioembolic risk determination beyond what we do at the moment.

Likely, the presence or absence of potentially thrombogenic "atrial disease" in the future will be the starting point for the stratification of cardioembolic risk and not the simple diagnosis of atrial fibrillation, which should lead to the development of new risk scores that include in their scope "atrial structure and function".

\section{REFERENCES}

1. Daley R, Mattingly TW, Holt CL, Bland EF, White PD. Systemic arterial embolism in rheumatic heart disease. Am Heart J. 1951;42(4):566-81. https://doi.org/10.1016/00028703(51)90152-4
2. Wolf PA, Dawber TR, Thomas E, Thomas Junior HE, Kannel WB. Epidemiologic assessment of chronic atrial fibrillation and risk of stroke: The Framingham Study. Neurology. 1978;28(10):973-7. https://doi.org/10.1212/wnl.28.10.973 
3. Wolf PA, Abbott RD, Kannel WB. Atrial fibrillation: a major contributor to stroke in the elderly. The Framingham Study. Arch Intern Med. 1987;147(9):1561-4.

4. Thygesen SK, Frost L, Eagle KA, Johnsen SP. Atrial fibrillation in patients with ischemic stroke: A population-based study. Clin Epidemiol. 2009;1:55-65. https://doi.org/10.2147/clep.s4794

5. Hannon N, Sheehan O, Kelly L, Marnane M, Merwick A, Moore A, et al. Stroke associated with atrial fibrillationincidence and early outcomes in the north Dublin population stroke study. Cerebrovasc Dis. 2010;29(1):43-9. https://doi. org/10.1159/000255973

6. Björck S, Palaszewski B, Friberg L, Bergfeldt L. Atrial fibrillation, stroke risk, and warfarin therapy revisited: a population-based study. Stroke. 2013;44(11):3103-8. https://doi.org/10.1161/ STROKEAHA.113.002329

7. Friberg L, Rosenqvist $M$, Lindgren A, Terént A, Norrving B, Asplund K. High prevalence of atrial fibrillation among patients with ischemic stroke. Stroke. 2014;45(9):2599-605. https://doi. org/10.1161/STROKEAHA.114.006070

8. Kishore A, Vail A, Majid A, Dawson J, Lees KR, Tyrrell PJ, et al. Detection of atrial fibrillation after ischemic stroke or transient ischemic attack - A systematic review and metaanalysis. Stroke. 2014;45(2):520-6. https://doi.org/10.1161/ STROKEAHA.113.003433

9. Gage BF, Waterman AD, Shannon W, Boechler M, Rich MW, Radford MJ. Validation of clinical classification schemes for predicting stroke. Results from the national registry of atrial fibrillation. JAMA. 2001;285(22):2864-70. https://doi. org/10.1001/jama.285.22.2864

10. Lip GYH, Nieuwlaat R, Pisters R, Lane DA, Crijns HJ. Refining clinical risk stratification for predicting stroke and thromboembolism in atrial fibrillation using a novel risk factor-based approach. The euro heart survey on atrial fibrillation. CHEST. 2010;137(2):26372. https://doi.org/10.1378/chest.09-1584

11. Singer DE, Chang Y, Borowsky LH, Fang MC, Pomernacki NK, Udaltsovaet $N$, et al. A new risk scheme to predict ischemic stroke and other thromboembolism in atrial fibrillation: The ATRIA study stroke risk score. J Am Heart Assoc. 2013;2(3):1-10. https://doi.org/10.1161/JAHA.113.000250

12. Wolsk E, Lamberts M, Morten LH, Blanche P, Køber L, Torp-Pedersen C, et al. Thromboembolic risk stratification of patients hospitalized with heart failure in sinus rhythm: a nationwide cohort study. Eur J Heart Fail. 2015;17(8):828-36. https://doi.org/10.1002/ejhf.309

13. Mazzone C, Cioffi G, Carriere C, Barbati G, Faganello G, Russo G, et al. Predictive role of CHA2DS2-VASc score for cardiovascular events and death in patients with arterial hypertension and stable sinus rhythm. Eur J Prev Cardiol. 2017;24(15):1584-93 https://doi.org/10.1177/2047487317726068

14. Guichard JB, Nattel S. Atrial cardiomyopathy: A useful notion in cardiac disease management or a passing fad? J Am Coll Cardiol. 2017;70:756-65. https://doi.org/10.1016/j.jacc.2017.06.033

15. Kottkamp H. Fibrotic atrial cardiomyopathy: A specific disease/ syndrome supplying substrates for atrial fibrillation, atrial tachycardia, sinus nodedisease, AVnodedisease, and thromboembolic complications. J Cardiovasc Electrophysiol. 2012;23(7):789-99. https:// doi.org/10.1111/j.1540-8167.2012.02341.x

16. Goette A, Kalman JM, Aguinaga L, Akar J, Cabrera JA, Chen SA, et al. EHRA/HRS/APHRS/SOLAECE expert consensus on atrial cardiomyopathies: definition, characterization, and clinical implication. Europace. 2016;18(10):1455-90. https://doi. org/10.1093/europace/euw161

17. Froehlich L, Meyre P, Aeschbacher S, Blum S, Djokic D, Kuehne $\mathrm{M}$, et al. Left atrial dimension and cardiovascular outcomes in patients with and without atrial fibrillation: a systematic review and meta-analysis. Heart. 2019;105(24):1884-91. https://doi. org/10.1136/heartjnl-2019-315174

18. Daccarett M, Badger TJ, Akoum N, Burgon NS, Mahnkopf C, Vergara $G$, et al. Association of left atrial fibrosis detected by delayed-enhancement magnetic resonance imaging and the risk of stroke in patients with atrial fibrillation. J Am Coll Cardiol. 2011;57(7):831-8. https://doi.org/10.1016/j.jacc.2010.09.049

19. Akoum N, Fernandez G, Wilson B, Mcgann C, Kholmovski E, Marrouche N. Association of atrial fibrosis quantified using LGE-MRI with atrial appendage thrombus and spontaneous contrast on transesophageal echocardiography in patients with atrial fibrillation.J Cardiovasc Electrophysiol. 2013;24(10):1104-9. https://doi.org/10.1111/jce.12199

20. King JB, Azadani PN, Suksaranjit P, Bress AP, Witt DM, Han $\mathrm{FT}$, et al. Left atrial fibrosis and risk of cerebrovascular and cardiovascular events in patients with atrial fibrillation. J Am Coll Cardiol. 2017;70(11):1311-21. https://doi.org/10.1016/j. jacc.2017.07.758

21. Müller $P$, Makimoto $H$, Dietrich JW, Fochler $F$, Nentwich $K$, Krug J, et al. Association of left atrial low-voltage area and thromboembolic risk in patients with atrial fibrillation. Europace. 2018;20(3):f359-65. https://doi.org/10.1093/europace/eux172

22. Glotzer TV, Daoud EG, Wyse DG, Singer DE, Ezekowitz MD, Hilker C, et al: The relationship between daily atrial tachyarrhythmia burden from implantable device diagnostics and stroke risk: the TRENDS study. Circ Arrhythm Electrophysiol. 2009;2(5):474-80. https://doi.org/10.1161/CIRCEP.109.849638

23. Daoud EG, Glotzer TV, Wyse DG, Ezekowitz MD, Hilker C, Koehler J, et al:Temporal relationship of atrial tachyarrhythmias, cerebrovascular events, and systemic emboli based on stored device data: A subgroup analysis of TRENDS. Heart Rhythm. 2011;8(9):1416-23. https://doi.org/10.1016/j.hrthm.2011.04.022

24. Brambatti M, Connolly SJ, Gold MR, Morillo CA, Capucci A, Muto C, et al: Temporal relationship between subclinical ttrial fibrillation and embolic events. Circulation. 2014;129(21):2094-99. https:// doi.org/10.1161/CIRCULATIONAHA.113.007825

25. Martin DT, Bersohn MM, Waldo AL, Wathen MS, Choucair WK, Lip GY, et al: Randomized trial of atrial arrhythmia monitoring to guide anticoagulation in patients with implanted defibrillator and cardiac resynchronization devices. Eur HeartJ. 2015;36(26):16608. https://doi.org/10.1093/eurheartj/ehv115

26. Mahajan R, Perera T, Elliott AD, Twomey DJ, Kumar S, Munwar $D A$, et al: Subclinical device-detected atrial fibrillation and stroke risk: a systematic review and meta-analysis. Eur Heart J. 2018;39(16):1407-15. https://doi.org/10.1093/eurheartj/ehx731 